\title{
Dynamical fermion mass generation by a strong Yukawa interaction
}

\author{
Tomáš Brauner ${ }^{1,2, \text { * } \text { and Jiří Hošek }}{ }^{1}$ \\ ${ }^{1}$ Department of Theoretical Physics, Nuclear Physics Institute, Řě̌, Czech Republic \\ ${ }^{2}$ Faculty of Mathematics and Physics, Charles University, Prague, Czech Republic
}

\begin{abstract}
We consider a model with global Abelian chiral symmetry of two massless fermion fields interacting with a complex massive scalar field. We argue that the Schwinger-Dyson equations for the fermion and boson propagators admit ultraviolet-finite chiral-symmetry-breaking solutions provided the Yukawa couplings are large enough. The fermions acquire masses and the elementary excitations of the complex scalar field are the two real spin-zero particles with different masses. As a necessary consequence of the dynamical chiral symmetry breakdown both in the fermion and scalar sectors, one massless pseudoscalar Nambu-Goldstone boson appears in the spectrum as a collective excitation of both the fermion and the boson fields. Its effective couplings to the fermion and boson fields are calculable.
\end{abstract}

PACS numbers: $11.30 . Q \mathrm{c}$

\section{INTRODUCTION}

One of the major challenges to current high energy physics is to understand the origin of particle masses [1]. All particle interactions except gravity are, up to energies accessible in to-date experiments, successfully described by the Standard model. There is therefore no doubt that the Standard model is the correct effective theory of particle interactions in the energy range so far explored.

It is well known that the detailed underlying physics manifests itself in the effective theory through the effective coupling constants so that when looking for new physics, we should try to reveal the origin of the parameters in the effective Lagrangian.

In the Standard model, the part of the Lagrangian describing the particle interactions is beautifully constrained by the gauge invariance principle. The matter part, however, seems rather ugly. The fermion masses cannot be introduced directly as they are prohibited by the gauge $S U(2)_{L} \times U(1)_{Y}$ symmetry, whose chiral structure is in turn necessary to provide an explanation for parity violation in weak interactions.

The masses therefore have to be generated by means of spontaneous symmetry breaking. This is achieved by introducing the scalar Higgs field and properly adjusting its potential so that it develops a non-zero vacuum expectation value at tree level. However, the dynamical origin of the 'wrong sign' of the Higgs mass squared, which is responsible for the tree-level condensation, remains unclear. Moreover, the fermion masses eventually stem from the Yukawa interaction with the Higgs, and hence there are as many different couplings as there are particle species. It would certainly be desirable to understand the particle masses as the consequence of some, yet unknown, quantum dynamics.

We would like to argue that dynamical mass generation is possible by means of the Yukawa interaction itself,

*Electronic address: brauner@ujf.cas.cz without ever having to change the sign of the Higgs mass squared. This would open a new possibility of the existence of an elementary massive scalar field in the Standard model Lagrangian. It should be heavy enough so that current experimental bounds are met, and also due to naturalness arguments, according to which its mass should be shifted by radiative corrections up to the scale of new physics.

Our ambitions in the present paper are much more modest than to cure the Standard model from its disease. We disregard the otherwise phenomenologically very important issues such as the hierarchy of particle families and $\mathrm{CP}$ violation, and concentrate on a simple Abelian model to show that spontaneous chiral symmetry breaking by the Yukawa interaction is viable. We believe that the central idea of the symmetry-breaking mechanism can thus be displayed more transparently. The implementation of this idea to the Standard model phenomenology is deferred to future work.

The plan of the paper is as follows. In the next section we introduce our model and investigate, at a rather elementary and pictorial level, the consequences of the assumed spontaneous generation of the fermion mass. We do so for the reader's convenience and to emphasize the robustness of the main idea of the paper. In particular we show that the fermion mass induces an anomalous symmetry-breaking two-point Green's function of the scalar. The spectrum of the system then contains two real spinless particles coupled to the original complex field, and with their masses split.

In the next part of the text a matrix formalism is developed which allows us to treat both the symmetrypreserving and the symmetry-breaking Green's functions on the same footing. We write down the one-loop Schwinger-Dyson equations and exploit the underlying symmetry by means of the Ward identities. Still assuming the spontaneous symmetry breaking to occur in the ground state, we show how the Nambu-Goldstone boson arises and examine its properties.

In the last part we demonstrate that, under reasonable simplifying assumptions a symmetry-breaking solution to 
the Schwinger-Dyson equations actually does exist, thus establishing a firm ground for the preceding heuristic arguments. We conclude the paper with a discussion of our results and the future perspective.

\section{PRELIMINARY CONSIDERATIONS}

Our model is defined by the Lagrangian,

$$
\begin{aligned}
\mathcal{L}=\sum_{j=1,2}\left(\bar{\psi}_{j L} i \not \partial \psi_{j L}+\bar{\psi}_{j R} i \not \partial \psi_{j R}\right)+ & \\
+\partial_{\mu} \phi^{\dagger} \partial^{\mu} \phi & -M^{2} \phi^{\dagger} \phi-\frac{1}{2} \lambda\left(\phi^{\dagger} \phi\right)^{2}+ \\
& +y_{1} \bar{\psi}_{1 L} \psi_{1 R} \phi+y_{1} \bar{\psi}_{1 R} \psi_{1 L} \phi^{\dagger}+ \\
& +y_{2} \bar{\psi}_{2 R} \psi_{2 L} \phi+y_{2} \bar{\psi}_{2 L} \psi_{2 R} \phi^{\dagger}
\end{aligned}
$$

The Yukawa couplings $y_{1,2}$ are real without lack of generality. Another remark is in order here. In view of the future application of our idea on the electroweak symmetry breaking, it is necessary that the global symmetry to be spontaneously broken is amenable to gauging. With just a single fermion species there would be an axial anomaly present. While anomaly cancelation is automatic in the Standard model due to its particle content, here we have to introduce two fermions with opposite axial charges to remove the anomaly in the Abelian axial current. It should be clear, however, that this minor technical complication does not alter at all the underlying idea.

The Lagrangian Eq. (11) enjoys a global Abelian $U(1)_{V 1} \times U(1)_{V 2} \times U(1)_{A}$ symmetry. The two vector $U(1)$ 's are associated with separate conservation of the numbers of fermions of the first and second type. The corresponding Noether currents are the well known

$$
\begin{aligned}
& j_{V 1}^{\mu}=\bar{\psi}_{1} \gamma^{\mu} \psi_{1}, \\
& j_{V 2}^{\mu}=\bar{\psi}_{2} \gamma^{\mu} \psi_{2} .
\end{aligned}
$$

The axial $U(1)$ transformations of the fermions are tied by the Yukawa coupling to the scalar field. For the Lagrangian to be invariant, it is necessary that the fields transform as

$$
\begin{aligned}
\psi_{1} & \rightarrow e^{+i \theta \gamma_{5}} \psi_{1}, \\
\psi_{2} & \rightarrow e^{-i \theta \gamma_{5}} \psi_{2}, \\
\phi & \rightarrow e^{-2 i \theta} \phi .
\end{aligned}
$$

The axial Noether current has the following form,

$$
j_{A}^{\mu}=\bar{\psi}_{1} \gamma^{\mu} \gamma_{5} \psi_{1}-\bar{\psi}_{2} \gamma^{\mu} \gamma_{5} \psi_{2}+2 i\left[\left(\partial^{\mu} \phi\right)^{\dagger} \phi-\phi^{\dagger} \partial^{\mu} \phi\right] .
$$

Now the standard tree-level mechanism of spontaneous symmetry breaking corresponds to $M^{2}<0$ in Eq. (11). Then the scalar field, $\phi=\frac{1}{\sqrt{2}}\left(\phi_{1}+i \phi_{2}\right)$, develops a non-zero ground-state expectation value, which is conveniently chosen to be real, $v \equiv\left\langle\phi_{1}\right\rangle_{0}=\left(-2 M^{2} / \lambda\right)^{1 / 2}$. Consequently, the fermions acquire the masses $m_{1,2}=$

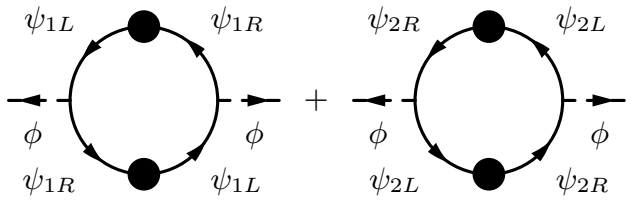

FIG. 1: Mixing in scalar sector induced by the fermion mass terms. The full blobs denote the chirality-changing part of the complete fermion propagator.

$\frac{1}{\sqrt{2}} v y_{1,2}, \phi_{2}$ becomes the massless Nambu-Goldstone boson, and $\phi_{1}^{\prime}=\phi_{1}-v$ becomes a massive real scalar particle with mass $M_{1}=\sqrt{-2 M^{2}}$. The Goldstone boson $\phi_{2}$ interacts with the fermions by the Yukawa coupling $\frac{m_{1}}{v} \bar{\psi}_{1} i \gamma_{5} \psi_{1} \phi_{2}-\frac{m_{2}}{v} \bar{\psi}_{2} i \gamma_{5} \psi_{2} \phi_{2}$.

From now on we set $M^{2}>0$ and investigate the model of Eq. (11) with respect to the possibility of spontaneous symmetry breaking. The scalar field now possesses an ordinary mass term, and there is therefore no condensation and no symmetry breaking at tree level. Rather, if the symmetry is broken in the ground state, it must be a result of the quantum dynamics of the system.

As spontaneous symmetry breaking is a nonperturbative phenomenon, it cannot be achieved at any finite order of perturbation theory. The non-perturbative method we employ here is to look for self-consistent symmetry-breaking solutions to the Schwinger-Dyson equations. We thus temporarily assume that there is a solution for which the full fermion propagators have non-vanishing chirality-changing parts, which means non-zero masses of the fermions.

Now let us observe that such a solution induces mixing of $\phi$ and $\phi^{\dagger}$ or, in other words, non-zero correlation function $\langle\phi \phi\rangle$, see Fig. 1 This could have been expected as the field $\phi$ couples to the axial part of the $U(1)_{V 1} \times U(1)_{V 2} \times U(1)_{A}$ symmetry, and once this is broken nothing prevents $\phi$ and $\phi^{\dagger}$ from mixing.

The second observation is that the 'anomalous' twopoint scalar Green's function $\langle\phi \phi\rangle$ in turn enters the oneloop Schwinger-Dyson equations for the fermion propagators, see Fig. 22 The set of Schwinger-Dyson equations for the fermion and scalar propagators are thus intrinsically coupled and must be solved simultaneously if a symmetry breaking solution is to be found.

Before we switch to a formal description to come in the next section, we would like to give a more physical picture of what is going on here. Let us for simplicity suppose that the one-particle-irreducible part of the anomalous scalar two-point function $\langle\phi \phi\rangle$ is momentum independent and equal to $-i \mu^{2}$. The spectrum in the scalar sector is then determined by the quadratic part of the renormalized Lagrangian,

$$
\mathcal{L}_{\text {scalar }}^{(0)}=\partial_{\mu} \phi^{\dagger} \partial^{\mu} \phi-M^{2} \phi^{\dagger} \phi-\frac{1}{2} \mu^{2 *} \phi \phi-\frac{1}{2} \mu^{2} \phi^{\dagger} \phi^{\dagger} .
$$

The non-derivative (mass) part of the Lagrangian is easily diagonalized by a rotation in the $\phi-\phi^{\dagger}$ space. 


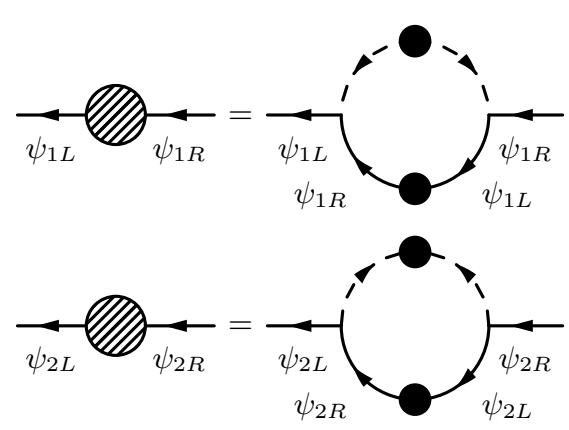

FIG. 2: Chirality changing fermion proper self-energies induced by the scalar mixing.

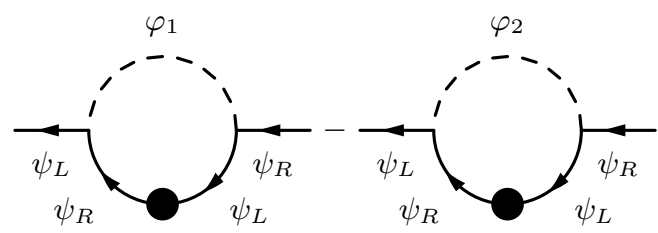

FIG. 3: One-loop SD equation for the fermion self-energy expressed in terms of the physical fields $\varphi_{1}$ and $\varphi_{2}$.

The spectrum then contains two real spin-0 particles with masses

$$
M_{1,2}^{2}=M^{2} \pm\left|\mu^{2}\right|
$$

The corresponding real fields $\varphi_{1}$ and $\varphi_{2}$ are defined through

$$
\phi=\frac{1}{\sqrt{2}} e^{i \alpha}\left(\varphi_{1}+i \varphi_{2}\right)
$$

where the 'mixing angle' $\alpha$ is merely given by the phase of the anomalous mass term, $\tan 2 \alpha=\operatorname{Im} \mu^{2} / \operatorname{Re} \mu^{2}$.

Note also that the anomalous propagator $\langle\phi \phi\rangle$ is now equal to $\frac{1}{2} e^{2 i \alpha}\left(\left\langle\varphi_{1} \varphi_{1}\right\rangle-\left\langle\varphi_{2} \varphi_{2}\right\rangle\right)$ that is, the one-loop graphs in Fig. 2 may be replaced with Fig. 3 The difference of scalar propagators significantly improves the convergence of the Schwinger-Dyson kernel.

\section{FORMAL DEVELOPMENTS}

\section{A. The scalar Nambu doublet}

We have seen in the previous section that once chiral symmetry is spontaneously broken the scalar field $\phi$ develops non-zero anomalous propagator mixing it with $\phi^{\dagger}$. For sake of simplicity of the general formulas to come, we introduce a doublet field

$$
\Phi=\left(\begin{array}{c}
\phi \\
\phi^{\dagger}
\end{array}\right)
$$

and the matrix propagator which contains as its entries both normal and anomalous two-point functions of the field $\phi$,

$$
\begin{aligned}
& i D(x-y)=\left\langle 0\left|T\left\{\Phi(x) \Phi^{\dagger}(y)\right\}\right| 0\right\rangle= \\
& \quad=\left(\begin{array}{cc}
\left\langle 0\left|T\left\{\phi(x) \phi^{\dagger}(y)\right\}\right| 0\right\rangle & \langle 0|T\{\phi(x) \phi(y)\}| 0\rangle \\
\left\langle 0\left|T\left\{\phi^{\dagger}(x) \phi^{\dagger}(y)\right\}\right| 0\right\rangle & \left\langle 0\left|T\left\{\phi^{\dagger}(x) \phi(y)\right\}\right| 0\right\rangle
\end{array}\right) .
\end{aligned}
$$

Note that this notation is similar to the Nambu formalism frequently used in the theory of superconductivity [2]. It is also quite natural due to its analogy in the fermion sector. There, we could have well introduced two 'normal' propagators for the left- and right-handed chiral fields and treat the desired mass term connecting $\psi_{L}$ with $\psi_{R}$ as an 'anomalous' part of the propagator. Instead, we work with the Dirac field

$$
\psi=\left(\begin{array}{c}
\psi_{L} \\
\psi_{R}
\end{array}\right)
$$

which incorporates both chiral fields in a single fourcomponent spinor. The chirality-changing part of the fermion propagator is then given by the off-diagonal component of the full Dirac propagator [12],

$$
\begin{aligned}
& i S(x-y)=\langle 0|T\{\psi(x) \bar{\psi}(y)\}| 0\rangle= \\
& =\left(\begin{array}{cc}
\left\langle 0\left|T\left\{\psi_{L}(x) \bar{\psi}_{L}(y)\right\}\right| 0\right\rangle & \left\langle 0\left|T\left\{\psi_{L}(x) \bar{\psi}_{R}(y)\right\}\right| 0\right\rangle \\
\left\langle 0\left|T\left\{\psi_{R}(x) \bar{\psi}_{L}(y)\right\}\right| 0\right\rangle & \left\langle 0\left|T\left\{\psi_{R}(x) \bar{\psi}_{R}(y)\right\}\right| 0\right\rangle
\end{array}\right) .
\end{aligned}
$$

The propagators of $\psi_{1}$ and $\psi_{2}$ will be denoted as $S_{1}$ and $S_{2}$, respectively.

\section{B. The Schwinger-Dyson equations}

As already stressed above, spontaneous symmetry breaking cannot be revealed at any finite order of perturbation theory. In order to deal with this non-perturbative effect, we employ the technique of the Schwinger-Dyson equations.

These constitute an infinite system of coupled equations for the Green's functions of the theory. To make them more tractable, it is usual to close the system at a certain order by assuming a convenient ansatz for the higher-point Green's functions. In order to achieve a simple gap equation we neglect all but the two-point connected Green's functions 3]. We are thus left with a selfconsistent set of equations for the fermion and scalar propagators, which are depicted diagrammatically in Fig. 4. The double-dashed line represents the Nambu doublet $\Phi$. This symbolic notation is used to stress the fact that the Schwinger-Dyson equations for both the symmetrypreserving and the symmetry-breaking parts of the propagators are represented by Feynman graphs of the same topology and can thus be put in a simple compact form as in Fig. 目.

We do not write down all the formulas that correspond to the Feynman diagrams in Fig. 4 Instead, having in mind our future simplification neglecting all symmetrypreserving radiative corrections, we put explicitly just 
the expressions for the symmetry-breaking proper selfenergies. The upper indices, $L$ or $R$ for the fermion propagators and 1 or 2 for the scalar propagator, specify the matrix elements of the two-by-two matrices for $S$ and $D$ introduced above. The same matrix notation is used for the proper self-energies $\Sigma$ and $\Pi$.

$$
\begin{aligned}
& \Sigma_{1}^{L R}(p)=i y_{1}^{2} \int \frac{d^{4} k}{(2 \pi)^{4}} S_{1}^{R L}(k) D^{12}(k-p) \\
& \Sigma_{2}^{L R}(p)=i y_{2}^{2} \int \frac{d^{4} k}{(2 \pi)^{4}} S_{2}^{R L}(k) D^{21}(k-p) \\
& \Pi^{12}(p)=-i y_{1}^{2} \int \frac{d^{4} k}{(2 \pi)^{4}} \operatorname{Tr}\left[S_{1}^{L R}(k) S_{1}^{L R}(k-p)\right]-i y_{2}^{2} \int \frac{d^{4} k}{(2 \pi)^{4}} \operatorname{Tr}\left[S_{2}^{R L}(k) S_{2}^{R L}(k-p)\right]+i \lambda \int \frac{d^{4} k}{(2 \pi)^{4}} D^{12}(k)
\end{aligned}
$$

Before concluding the discussion of the SchwingerDyson equations, let us note that neglecting corrections to the interaction vertices is not that all consistent with the envisaged spontaneous symmetry breaking. In Sec. IIIC we explain that the coupling of both the fermions and the scalar to the current of the broken symmetry (that is, the axial current) develops a pole due to the intermediate massless Nambu-Goldstone boson state. Now the same is also true for the (pseudo)scalar Yukawa coupling of the fermion and scalar. As a result, the full $\Phi$ propagator should possess a massless pole due to the Nambu-Goldstone boson, with the residuum determined by the factor $\langle 0|\phi| \pi(q)\rangle$.

Neglecting the vertex corrections therefore means that we are not going to reproduce correctly the whole analytical structure of the propagators. Our set of SchwingerDyson equations amounts to resummation of a certain class of Feynman diagrams which, however, is sufficient to discover spontaneous symmetry breaking. In other words, we are looking for spontaneous symmetry breaking in the spectrum of the elementary excitations of the fields $\psi_{1,2}$ and $\Phi$. The discussion of the collective excitations, which of course also manifest themselves in the full propagators, is deferred to Sec. IIIC

\section{The Ward identities}

We now exploit the symmetry properties of the theory. At the classical level, the $U(1)_{V 1} \times U(1)_{V 2} \times U(1)_{A}$ invariance of the Lagrangian (1) implies the existence of three conserved Noether currents - two vector, see Eq. (2), and one axial, see Eq. (4).

At the level of quantum field theory, the conservation of the vector and axial currents is expressed in terms of the set of Ward identities for the Green's functions containing the current operators. We investigate here the three-point functions with the conserved current and a fermion or scalar pair, respectively.

The vector currents couple only to the fermions, so there is just one non-trivial Ward

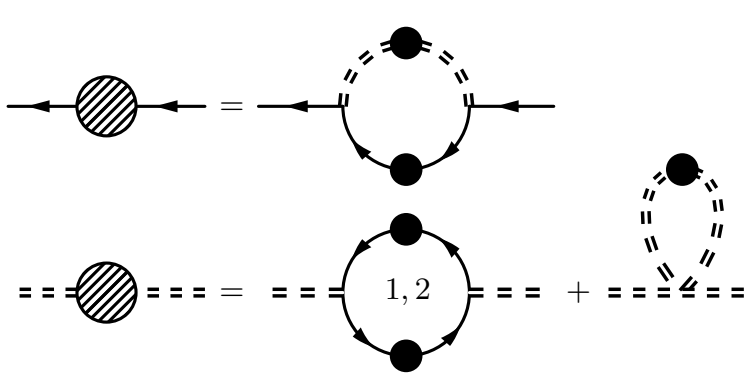

FIG. 4: The diagrammatical representation of the one-loop Schwinger-Dyson equations for the fermion and scalar selfenergies. The first line holds for both $\psi_{1}$ and $\psi_{2}$. The dashed blobs stand for the proper self-energies, while the solid blobs denote the full propagators. The double dashed line is for the Nambu $\Phi$ doublet.

identity for each, for the vertex functions $G_{V 1}^{\mu}(x, y, z)=\left\langle 0\left|T\left\{j_{V 1}^{\mu}(x) \psi_{1}(\underline{y}) \bar{\psi}_{1}(z)\right\}\right| 0\right\rangle \quad$ and $G_{V 2}^{\mu}(x, y, z)=\left\langle 0\left|T\left\{j_{V 2}^{\mu}(x) \psi_{2}(y) \bar{\psi}_{2}(z)\right\}\right| 0\right\rangle$, which have the well known form

$$
\begin{aligned}
& q_{\mu} \Gamma_{V 1}^{\mu}(p+q, p)=S_{1}^{-1}(p+q)-S_{1}^{-1}(p) \\
& q_{\mu} \Gamma_{V 2}^{\mu}(p+q, p)=S_{2}^{-1}(p+q)-S_{2}^{-1}(p) .
\end{aligned}
$$

The proper vertex functions $\Gamma_{V 1,2}^{\mu}$ correspond to $G_{V 1,2}^{\mu}$ with full fermion propagators of the external legs cut off.

The axial current, on the other hand, couples to both the fermions and the scalar. There are hence altogether three vertex functions, $G_{A \psi_{1}}^{\mu}(x, y, z)=\left\langle 0\left|T\left\{j_{A}^{\mu}(x) \psi_{1}(y) \bar{\psi}_{1}(z)\right\}\right| 0\right\rangle$, $G_{A \psi_{2}}^{\mu}(x, y, z)=\left\langle 0\left|T\left\{j_{A}^{\mu}(x) \psi_{2}(y) \bar{\psi}_{2}(z)\right\}\right| 0\right\rangle$, and $G_{A \phi}^{\mu}(x, y, z)=\left\langle 0\left|T\left\{j_{A}^{\mu}(x) \Phi(y) \Phi^{\dagger}(z)\right\}\right| 0\right\rangle$. The corresponding Ward identities read

$$
\begin{aligned}
q_{\mu} \Gamma_{A \psi_{1}}^{\mu}(p+q, p) & =S_{1}^{-1}(p+q) \gamma_{5}+\gamma_{5} S_{1}^{-1}(p), \\
q_{\mu} \Gamma_{A \psi_{2}}^{\mu}(p+q, p) & =-S_{2}^{-1}(p+q) \gamma_{5}-\gamma_{5} S_{2}^{-1}(p), \\
q_{\mu} \Gamma_{A \phi}^{\mu}(p+q, p) & =-2 D^{-1}(p+q) \Xi+2 \Xi D^{-1}(p) .
\end{aligned}
$$


The matrix $\Xi$,

$$
\Xi=\left(\begin{array}{rr}
1 & 0 \\
0 & -1
\end{array}\right)
$$

operates in the $\phi-\phi^{\dagger}$ space and is quite analogous to $\gamma_{5}$ in the fermion sector.

Before closing the general discussion of the Ward identities, let us remark that the identities of Eq. (7) must hold whether the symmetry is spontaneously broken or not. In both cases they strongly constrain the form of the vertex functions, but particularly if the symmetry is broken, they allow us to visualize the massless collective excitation predicted by the Goldstone theorem, as we will now see.

\section{The Nambu-Goldstone boson}

Once chiral symmetry is broken, there must exist a massless Nambu-Goldstone boson in the spectrum of the theory, which couples to the Noether current of the broken symmetry. It is the axial current which is broken in our case, and as it couples to both the fermions and the scalar, the Nambu-Goldstone boson must be a collective excitation of both fermions and scalars.

General analytical properties of Green's functions imply the existence of a pole corresponding to an intermediate particle, once the total momentum of a proper subset of external legs approaches the mass shell of the particle [4]. This means that the Nambu-Goldstone boson can be seen as a pole in the vertex functions of the axial current as $q^{2} \rightarrow 0$. Its properties are then expressed in terms of the symmetry-breaking self-energies of the fermions and the scalar, which are obtained by solving the set of Schwinger-Dyson equations stated in Sec. IIIB

To proceed further, we approximate the proper vertex functions $\Gamma_{A \psi_{1,2}}^{\mu}$ and $\Gamma_{A \phi}^{\mu}$ by the sum of the bare vertex and the pole contribution. We follow the analysis of Jackiw and Johnson [5].

The bare vertices are determined by the usual rules of perturbation theory. For sake of later reference and to show how the $\Phi$ notation works, we fix them down in Fig. [5]

The pole of the vertex functions arises from the propagator of the intermediate Nambu-Goldstone boson, see Fig. [6] The yet unknown effective vertices of the NambuGoldstone boson with the fermions and the scalar, to be extracted from the Ward identities (7), are denoted by empty circles in the figures and by $P_{\psi_{1,2}}(p+q, p)$ and $P_{\phi}(p+q, p)$ in the formulas.

We can now write down the formulas for the pole con-

$$
\begin{gathered}
\Gamma_{A \psi_{1}, \text { bare }}^{\mu}(p+q, p)= \\
\Gamma_{A \psi_{2}, \text { bare }}^{\mu}(p+q, p)= \\
\Gamma_{A \phi, \text { bare }}^{\mu}(p+q, p)=\gamma^{\mu} \gamma_{5},
\end{gathered}
$$

FIG. 5: The bare parts of the proper vertex functions of the axial current. The crosses indicate the axial current. The numbers at the solid lines distinguish between $\psi_{1}$ and $\psi_{2}$.

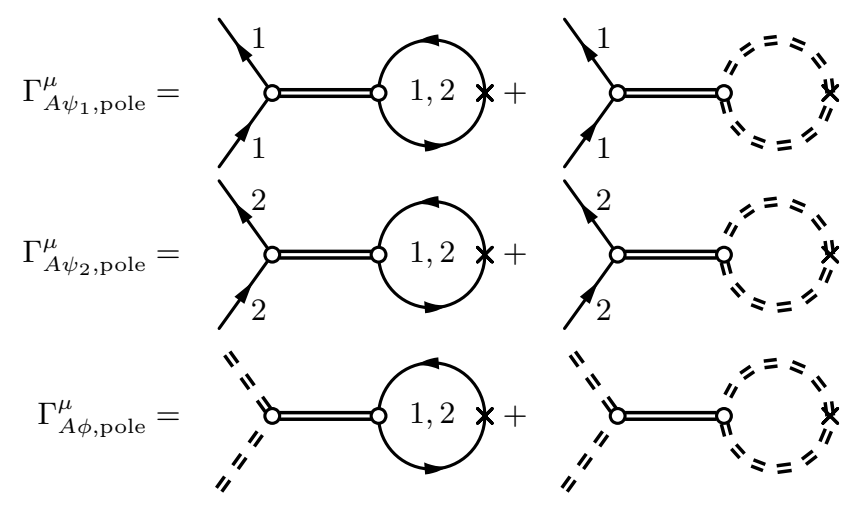

FIG. 6: The pole parts of the proper vertex functions of the axial current. The double solid line represents the NambuGoldstone boson and the empty circles its effective vertices with the fermions and the scalar, respectively. Both $\psi_{1}$ and $\psi_{2}$ can circulate in the closed fermion loops.

tributions,

$$
\begin{aligned}
\Gamma_{A \psi_{1}, \text { pole }}^{\mu} & =P_{\psi_{1}}(p+q, p) \frac{i}{q^{2}}\left[I_{\psi_{1}}^{\mu}(q)+I_{\psi_{2}}^{\mu}(q)+I_{\phi}^{\mu}(q)\right], \\
\Gamma_{A \psi_{2}, \text { pole }}^{\mu} & =P_{\psi_{2}}(p+q, p) \frac{i}{q^{2}}\left[I_{\psi_{1}}^{\mu}(q)+I_{\psi_{2}}^{\mu}(q)+I_{\phi}^{\mu}(q)\right], \\
\Gamma_{A \phi, \text { pole }}^{\mu} & =P_{\phi}(p+q, p) \frac{i}{q^{2}}\left[I_{\psi_{1}}^{\mu}(q)+I_{\psi_{2}}^{\mu}(q)+I_{\phi}^{\mu}(q)\right],
\end{aligned}
$$

where $I_{\psi_{1,2}}^{\mu}(q)$ and $I_{\phi}^{\mu}(q)$ represent the fermion and scalar loops in Fig. 6] They are given by the equations 


$$
\begin{aligned}
I_{\psi_{1}}^{\mu}(q) & =-\int \frac{d^{4} k}{(2 \pi)^{4}} \operatorname{Tr}\left[\gamma^{\mu} \gamma_{5} i S_{1}(k-q) P_{\psi_{1}}(k-q, k) i S_{1}(k)\right] \\
I_{\psi_{2}}^{\mu}(q) & =-\int \frac{d^{4} k}{(2 \pi)^{4}} \operatorname{Tr}\left[-\gamma^{\mu} \gamma_{5} i S_{2}(k-q) P_{\psi_{2}}(k-q, k) i S_{2}(k)\right] \\
I_{\phi}^{\mu}(q) & =\frac{1}{2} \int \frac{d^{4} k}{(2 \pi)^{4}} \operatorname{Tr}\left[-2(2 k-q)^{\mu} \Xi i D(k-q) P_{\phi}(k-q, k) i D(k)\right] .
\end{aligned}
$$

The difference of the integral prefactors here arises from the different nature of the degrees of freedom circulating in the loops. The -1 is the standard fermion loop factor, while the $\frac{1}{2}$ follows from the fact that by introducing $\Phi$ instead of $\phi$ we have effectively doubled the number of degrees of freedom, which must be compensated for when calculating the trace over a closed loop.

As the loop integrals $I_{\psi_{1,2}}^{\mu}(q)$ and $I_{\phi}^{\mu}(q)$ depend only on a single external momentum $q$, they are forced by Lorentz covariance to have the form

$$
\begin{gathered}
I_{\psi_{1,2}}^{\mu}(q)=-i q^{\mu} I_{\psi_{1,2}}\left(q^{2}\right), \\
I_{\phi}^{\mu}(q)=-i q^{\mu} I_{\phi}\left(q^{2}\right) .
\end{gathered}
$$

Inserting this into Eq. (8) and the sum of the bare and the pole contributions into the Ward identities (7), and going onto the Nambu-Goldstone boson mass shell, $q^{2} \rightarrow 0$, we arrive at the general formula for the effective vertices,

$$
\begin{aligned}
P_{\psi_{1}}(p+q, p) & =\frac{1}{N}\left[S_{1}^{-1}(p+q) \gamma_{5}+\gamma_{5} S_{1}^{-1}(p)-q \gamma_{5}\right], \\
P_{\psi_{2}}(p+q, p) & =-\frac{1}{N}\left[S_{2}^{-1}(p+q) \gamma_{5}+\gamma_{5} S_{2}^{-1}(p)-q \gamma_{5}\right], \\
P_{\phi}(p+q, p) & =-\frac{2}{N}\left[D^{-1}(p+q) \Xi-\Xi D^{-1}(p)-q \cdot(2 p+q) \Xi\right],
\end{aligned}
$$

where the normalization factor $N$ is given by $N=$ $I_{\psi_{1}}(0)+I_{\psi_{2}}(0)+I_{\phi}(0)$. As noted by Jackiw and Johnson [5], the effective vertices found this way are ambiguous at the order $O\left(q^{2}\right)$, since we have approximated the loop corrections to the full proper vertex functions $\Gamma_{A \psi_{1,2}}^{\mu}$ and $\Gamma_{A \phi}^{\mu}$ by their pole parts, and completely neglected other finite contributions.

To complete the calculation of the vertex functions, we must now plug the expressions (10) back into Eq. (9) and solve the resulting system of equations for $I_{\psi_{1,2}}(0)$ and $I_{\phi}(0)$.

\section{MODEL RESULTS}

\section{A. Effective vertices and loop integrals}

The results obtained above are fairly general as we have used only very few and weak assumptions like the pole term dominance in the proper vertex functions. On the other hand, it is not easy to draw any concrete results from formulas like Eqs. (91) and (10). To push our conclusions little bit further, we now make a simplifying assumption that will allow us to finish the calculation.

Since we are looking for spontaneous symmetry breaking, we shall neglect ordinary (symmetry-preserving) renormalization of the fermion and scalar propagators [ 6 ] and retain just the symmetry-breaking self-energies. This will enable us to proceed analytically as far as possible. Of course, as soon as one pretends at phenomenological relevance of the obtained results, all radiative corrections must be included, but this is not the aim of the present paper. Here we just wish to demonstrate that spontaneous symmetry breaking is possible in a model like Eq. (1).

We thus make the following ansatz for the fermion and 
scalar propagators,

$$
\begin{aligned}
S_{1,2}^{-1}(p) & =\not p-\Sigma_{1,2}(p), \\
D^{-1}(p) & =\left(\begin{array}{cc}
p^{2}-M^{2} & -\Pi(p) \\
-\Pi^{*}(p) & p^{2}-M^{2}
\end{array}\right),
\end{aligned}
$$

where $\Sigma_{1,2}(p)$ are the Lorentz-scalar chirality-changing proper self-energies, and $\Pi(p)$ is the anomalous proper self-energy of the scalar field.

With this assumption, the effective vertices (10) become

$$
\begin{aligned}
& P_{\psi_{1}}(p+q, p)=-\frac{1}{N}\left[\Sigma_{1}(p+q)+\Sigma_{1}(p)\right] \gamma_{5}, \\
& P_{\psi_{2}}(p+q, p)=\frac{1}{N}\left[\Sigma_{2}(p+q)+\Sigma_{2}(p)\right] \gamma_{5}, \\
& P_{\phi}(p+q, p)=-\frac{2}{N}\left(\begin{array}{cc}
0 & \Pi(p+q)+\Pi(p) \\
-\Pi^{*}(p+q)-\Pi^{*}(p) & 0
\end{array}\right) .
\end{aligned}
$$

$$
\begin{aligned}
-i q^{\mu} J_{\psi_{1}}\left(q^{2}\right) & =8 \int \frac{d^{4} k}{(2 \pi)^{4}} \frac{(k-q)^{\mu} \Sigma_{1, k}}{k^{2}-\Sigma_{1, k}^{2}} \frac{\Sigma_{1, k}+\Sigma_{1, k-q}}{(k-q)^{2}-\Sigma_{1, k-q}^{2}}, \\
-i q^{\mu} J_{\psi_{2}}\left(q^{2}\right) & =8 \int \frac{d^{4} k}{(2 \pi)^{4}} \frac{(k-q)^{\mu} \Sigma_{2, k}}{k^{2}-\Sigma_{2, k}^{2}} \frac{\Sigma_{2, k}+\Sigma_{2, k-q}}{(k-q)^{2}-\Sigma_{2, k-q}^{2}}, \\
-i q^{\mu} J_{\phi}\left(q^{2}\right) & =8 \int \frac{d^{4} k}{(2 \pi)^{4}} \frac{(2 k-q)^{\mu}\left(k^{2}-M^{2}\right)}{\left(k^{2}-M^{2}\right)^{2}-\left|\Pi_{k}\right|^{2}} \frac{\operatorname{Re}\left[\Pi_{k-q}^{*}\left(\Pi_{k}+\Pi_{k-q}\right)\right]}{\left[(k-q)^{2}-M^{2}\right]^{2}-\left|\Pi_{k-q}\right|^{2}} .
\end{aligned}
$$

With these definitions, the expressions for $I_{\psi_{1,2}}\left(q^{2}\right)$ and $I_{\phi}\left(q^{2}\right) \mathrm{read}$

$$
\begin{aligned}
I_{\psi_{1,2}}\left(q^{2}\right) & =\frac{J_{\psi_{1,2}}\left(q^{2}\right)}{N}, \\
I_{\phi}\left(q^{2}\right) & =\frac{J_{\phi}\left(q^{2}\right)}{N},
\end{aligned}
$$

wherefrom the overall normalization factor of the vertices in Eq. (12) is equal to

$$
N=\sqrt{J_{\psi_{1}}(0)+J_{\psi_{2}}(0)+J_{\phi}(0)} .
$$

We can now go on to evaluate the last missing piece that is, the normalization factors $I_{\psi_{1,2}}(0)$ and $I_{\phi}(0)$. We substitute for the propagators (11) and the effective vertices (12) in the loop integrals (9), which turn out to be parameterized in terms of the integrals (for sake of readability we put the arguments of $\Sigma_{1,2}$ and $\Pi$ to the lower index),
Let us finally note that the ansatz (11) also allows us to simplify the Schwinger-Dyson equations (6), whose solution will be the subject of the next section. With the same index notation as above in Eq. (13), Eqs. (6) become

$$
\begin{aligned}
\Sigma_{1, p} & =i y_{1}^{2} \int \frac{d^{4} k}{(2 \pi)^{4}} \frac{\Sigma_{1, k}}{k^{2}-\Sigma_{1, k}^{2}} \frac{\Pi_{k-p}}{\left[(k-p)^{2}-M^{2}\right]^{2}-\left|\Pi_{k-p}\right|^{2}}, \\
\Sigma_{2, p} & =i y_{2}^{2} \int \frac{d^{4} k}{(2 \pi)^{4}} \frac{\Sigma_{2, k}}{k^{2}-\Sigma_{2, k}^{2}} \frac{\Pi_{k-p}^{*}}{\left[(k-p)^{2}-M^{2}\right]^{2}-\left|\Pi_{k-p}\right|^{2}}, \\
\Pi_{p} & =-\sum_{j=1,2} 2 i y_{j}^{2} \int \frac{d^{4} k}{(2 \pi)^{4}} \frac{\Sigma_{j, k}}{k^{2}-\Sigma_{j, k}^{2}} \frac{\Sigma_{j, k-p}}{(k-p)^{2}-\Sigma_{j, k-p}^{2}}+i \lambda \int \frac{d^{4} k}{(2 \pi)^{4}} \frac{\Pi_{k}}{\left(k^{2}-M^{2}\right)^{2}-\left|\Pi_{k}\right|^{2}} .
\end{aligned}
$$

\section{B. Solution to the Schwinger-Dyson equations}

We now wish to demonstrate that the SchwingerDyson equations (15) actually do have a non-trivial so- lution that is, our mechanism is capable of generating 


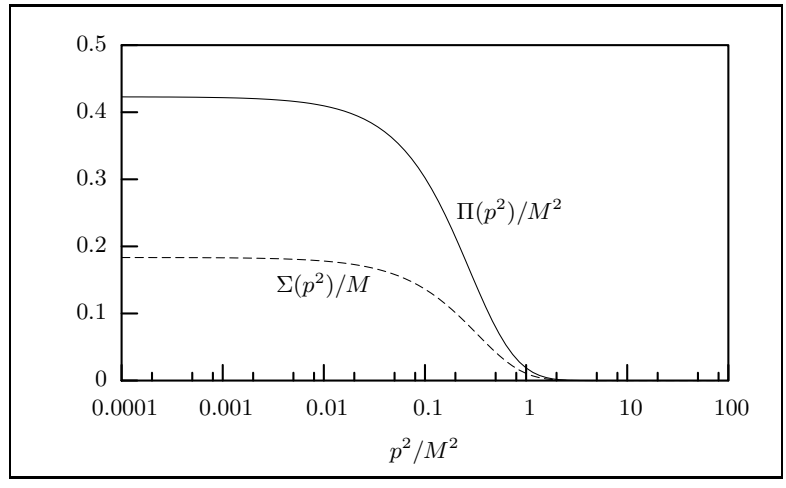

FIG. 7: Results of the numerical computation of the selfenergies $\Sigma$ and $\Pi$.

fermion masses dynamically. To that end note that Eqs. (15) constitute a set of coupled non-linear integral equations for the unknown functions $\Sigma_{1, p}, \Sigma_{2, p}$ and $\Pi_{p}$. This is still too complicated to deal with, and we therefore introduce further simplifications. We keep in mind that we are just attempting to break the chiral symmetry dynamically, and do not intend to produce any phenomenological conclusions at this stage.

First, we abandon the $\lambda$-term in the last of Eqs. (15). Formally, the advantage of this step is in the fact that $\Pi$ is then expressed exclusively in terms of the $\Sigma$ 's. Physically, the symmetry is broken by the strong dynamics of the Yukawa interaction, while the scalar field quartic selfinteraction can be switched on perturbatively later. This possibility is in marked contrast with the standard use of a condensing scalar. The $\lambda\left(\phi^{\dagger} \phi\right)$ term in the Lagrangian will become indispensable as a counter-term after including ordinary, symmetry-preserving quantum corrections.

Second, we consider for simplicity just the special case $y_{1}=y_{2}$. The Lagrangian is then invariant under the discrete symmetry $\psi_{1} \leftrightarrow \psi_{2}, \phi \leftrightarrow \phi^{\dagger}$. As far as the induced anomalous scalar self-energy is real, we may assume that this discrete symmetry is not spontaneously broken and the self-energies $\Sigma_{1}$ and $\Sigma_{2}$ are therefore equal. We are thus left with two coupled equations for $\Pi$ and just one $\Sigma$.

The reduced set of equations may now in principle be solved iteratively. We performed a numerical calculation to estimate the order of magnitude of the generated fermion mass. We used the Euclidean approximation that is, made a formal Wick rotation of the momenta in Eqs. (15). We made use of the fact that, after canceling the $\lambda$-term, the right hand side of the last of Eqs. (15) depends just on the $\Sigma$ 's.

We took an initial ansatz for the $\Sigma$ and used it to calculate the zeroth approximation for the $\Pi$. After then, we solved the two coupled equations iteratively. Our results are summarized by the graphs in Fig. 7

We can see that the Eqs. (15) indeed possess a nontrivial solution. As far as we were able to check, this solution is unique in the sense that it is independent of the initial ansatz for the $\Sigma^{\prime} s$. The self-energies fall down rapidly once the momentum exceeds the bare scalar mass $M$, thus verifying our assumptions on the convergence of the loop integrals.

It would be perhaps more appropriate to do all the calculations in the Minkowski space as the physical mass lies in the time-like region of momenta. Such a calculation has been performed in Ref. [7].

\section{DISCUSSION AND CONCLUSION}

Within simplifying assumptions stated in the text we have demonstrated that a strong chirally invariant Yukawa interaction of massless fermions with a massive complex scalar field can generate the fermion masses by genuinely quantum (i.e. non-perturbative) loop effects. By the existence theorem this implies the massless pseudoscalar Nambu-Goldstone boson in the spectrum. In this respect our program is very much the same as that of the renown NJL paper [8].

We believe that certain appeal of our suggestion is in the ultraviolet finiteness of non-perturbatively calculated quantities. It can be traced to the necessity of a generic coupling of the Schwinger-Dyson equations for the fermion and the scalar field propagators. It is definitely more subtle that the single Schwinger-Dyson equation for the fermion propagator with chirality conserving vector interactions. Technically the $\phi-\phi^{\dagger}$ mixing results in the difference of propagators of the scalar mass eigenstates and, consequently, in decent ultraviolet behavior of anomalous (symmetry-breaking) loop integrals.

Vexing assumptions of the present exploratory stage of the development of the model have to be replaced by better ones. In particular it is desirable to have approximate analytic solutions $\Sigma\left(p^{2}\right)$ and $\Pi\left(p^{2}\right)$ in Minkowski space. The fermion masses $m_{1,2}$ are determined by solving $m_{1,2}^{2}=\Sigma_{1,2}^{2}\left(p^{2}=m_{1,2}^{2}\right)$ and by the dimensional argument the solution must have the form

$$
m_{1,2}=M f_{1,2}\left(y_{1,2}\right) .
$$

Preliminary numerical analysis in Euclidean space suggests that $\Sigma_{1,2} \rightarrow 0$ for $y_{1,2}$ approaching a (large) critical value. Our numerical calculation gives a rough estimate $y_{\text {crit. }} \approx 35$. The formula (16) is to be compared with $m_{1,2}=\frac{1}{\sqrt{2}} v y_{1,2}$ of the standard tree-level approach with a condensing scalar.

Possible uses of our model, if harshly justified, are also those of NJL in its contemporary interpretation:

(i) The apparently non-BCS-like form of the Schwinger-Dyson equations for the fermion propagator (6) is suggestive for modeling fermionic superfluidity with scalar effective degrees of freedom.

(ii) Non-Abelian generalization and gauging of the NJL model resulted in the past in models of the dynamical mass generation in $S U(2)_{L} \times U(1)_{Y}$ gauge-invariant electroweak models [9, 10]. With our way of treating scalars we plan to follow the same path [11]. 


\section{Acknowledgments}

We are grateful to Petr Beneš for doing the numerical calculation of the self-energies. This work was supported in part by the Institutional Research Plan AV0Z10480505, and by the GACR doctoral project No. 202/05/H003.
[1] C. Quigg (2005), hep-ph/0502070.

[2] Y. Nambu, Phys. Rev. 117, 648 (1960).

[3] A. L. Fetter and J. D. Walecka, Quantum theory of manyparticle systems, International series in pure and applied physics (McGraw-Hill, New York, 1971).

[4] S. Weinberg, The Quantum Theory of Fields, vol. 1 (Cambridge University Press, Cambridge, 1995), 1st ed.

[5] R. Jackiw and K. Johnson, Phys. Rev. D8, 2386 (1973).

[6] J. M. Cornwall, R. Jackiw, and E. Tomboulis, Phys. Rev. D10, 2428 (1974).

[7] P. Bicudo, Phys. Rev. D69, 074003 (2004), hep$\mathrm{ph} / 0312373$.

[8] Y. Nambu and G. Jona-Lasinio, Phys. Rev. 122, 345
(1961).

[9] J. Hošek (1985), CERN-TH-4104/85.

[10] W. A. Bardeen, C. T. Hill, and M. Lindner, Phys. Rev. D41, 1647 (1990).

[11] T. Brauner and J. Hošek (2004), hep-ph/0407339.

[12] We work in the chiral basis of the Dirac $\gamma$-matrices, in which $\gamma_{5}$ is diagonal, and quite deliberately denote by $\psi_{L, R}$ both the two-component Weyl spinors and the fourcomponent Dirac spinors with just the upper two or the lower two entries non-zero. It should be clear from the context which of these two spinors is actually used. 